constant angular velocity $\alpha$, exerting the moment $m a$ upon the shelf $D$, and therefore on the car. ${ }^{1}$

This precession continues until the roller and the shelf cease to touch. At first $\mathrm{H}$ lifts with the shelf, and afterwards the shelf moves downwards, followed for some distance by the roller. If the tilt had been in the opposite direction, the shelf $\mathrm{D}$ would have acted upon the roller $\mathrm{H}^{\prime}$, and caused just the opposite kind of precession, and a moment of the opposite kind.

We now have the spindles out of their mid-positions as $\mathrm{OQ}, \mathrm{O}^{\prime} \mathrm{Q}^{\prime}$. How are they brought back to $\mathrm{NOO}^{\prime} \mathrm{N}^{\prime}$ with $\mathrm{H}$ permanently lowered?

It is the essence of Mr. Brennan's invention that, after a restoring moment has been applied to the car, the spindles shall go back to the position $\mathrm{NOO}^{\prime} \mathrm{N}^{\prime}$ with $\mathrm{H}$ permanently lowered, so as to be ready to act again,

$\mathrm{He}$ effects this object in various ways. Some ways described in his patents are quite different from what is used on the model, and the method to be used on the fullsize waggon will again be quite different. I will describe one of these methods. Mr. Brennan tells me that he considers this old method to be crude, but he is naturally unwilling to allow me to publish his latest method.

$D^{\prime}$ is a circular shelf extending from the mid-position in my direction; $D$ is a similar shelf extending from the mid-position into the paper or away from me. It is on these shelves that $\mathrm{H}^{\prime}$ and $\mathrm{H}$ roll, causing precession, as I have just described. When $\mathrm{H}^{\prime}$ is inside the paper or when $\mathrm{H}$ is outside the paper they find no shelf to roll upon. There are, however, two other shelves, $\mathrm{L}$ and $\mathrm{L}^{\prime}$, for two other rollers, $M$ and $M^{\prime}$, which are attached to the frames concentric with the spindles. They are free to rotate, but are not rotated by the spindles. When they are pressed by their shelves $L$ or $L^{\prime}$ this causes negative precession, and they roll towards the $\mathrm{NOO}^{\prime} \mathrm{N}^{\prime}$ position. There is, of course, friction at their supports retarding their rotation, and therefore the precession. The important thing to remember is that $\mathrm{H}$ and $\mathrm{H}^{\prime}$ when they touch their shelves (when one is touching, the other is not touching) cause a precession away from the mid-position at a rate $\alpha$ which produces a restoring moment $m \alpha$ of constant amount (except for slipping), whereas when $\mathrm{M}$ or $\mathrm{M}^{\prime}$ touches its shelf $\mathrm{L}$ or $\mathrm{I}^{\prime}$ (when one is touching the other is not touching), the pressure on the shelf and friction determine the rate of precession towards the mid-position as well as the small vertical motion.

Suppose the tilt to be corrected is $R$, when $D$ presses $\mathrm{H}$ upward. The moment $m a$ and its time of action (the total momental impulse) are too great, and $R$ is overrcorrected; this causes the rollers $M^{\prime}$ to act on $L^{\prime}$, and the spindles return to the mid-position; they go beyond the mid-position, and now the roller $\mathrm{H}^{\prime}$ acts on $\mathrm{D}^{\prime}$, and there is a return to the mid-position and beyond it, and so it goes on-the swings of the gyrostats out of and into the mid-position, and the vibrations of the car about its position of equilibrium getting rapidly less and less, until again neither $\mathrm{H}$ or $\mathrm{H}^{\prime}$ nor $\mathrm{M}$ or $\mathrm{M}^{\prime}$ is touching a shelf. It is indeed marvellous to see how rapidly the swings decay. ${ }^{2}$

It wili be seen that by using the two gyrostats instead of one, when there is a curve on the line, although the plane $\mathrm{NOO}^{\prime} \mathrm{N}^{\prime}$ rotates, and we may say that the gyrostats precess, the tilting couples which they exercise are equal and opposite.

It is evident that this method of Mr. Brennan is altogether different in character from that of $\mathrm{Mr}$. Schlick.

1 I am supposing the precessional angles to be small ; when the angles are like $N O Q, N^{\prime} O^{\prime} Q^{\prime}$. Fig. 3 , the sum of the moments of the two gyrostats like $\mathrm{NOQ}, \mathrm{N}^{\prime} \mathrm{O}^{\prime} \mathrm{Q}$. Fig. 3 , the sum of the moments of the two gyrostats
would be ma cos $\mathrm{N} \cap Q, a$ being constant. if there were no slipping; but there is alwavs slipping, and the gond working of the apparatus requires
that there shall be such slipping. $a$ is not constant, and it is always less than that there shall be such slipping. a is not cons
what it wonld be if there was true rolling.

1 If in Fig. $3 \mathrm{R}$ is the angle which the waggon makes with its position of
and If in Fig. $3 \mathrm{R}$ is the angle which the waggon makes with its position of
equilibrium; if $M$ is the moment with which the shelf $D$ acts clockwise equilibrium; if $M$ is the moment with which the shelf $D$ acts clockwise
upon $H$, and $P$ is the angle of precession $Q \circ V_{\text {; }}$ ard if $\mu$ is the coefficient upon $\mathrm{H}$, and $\mathrm{P}$ is the angle of precession $\mathrm{Q} \circ \mathrm{V}$; and if $\mu$ is the coefficient
of friction between $\mathrm{D}$ and $\mathrm{H}$, then in the first part of the action above of friction hetween $\mathrm{D}$ and $\mathrm{H}$, than in the first part of the action above
described $\mathrm{I} \theta^{2} \mathrm{R}+m \theta \mathrm{P}-\mathrm{M}=0 . m \theta \mathrm{R}-i \theta^{2} \mathrm{P}+\mu \mathrm{M}=0,\left(\mathrm{I}_{1} \theta^{2}-h w\right) \mathrm{R}+\mathrm{M}=0$, if described $\mathrm{I} \theta^{2} \mathrm{R}+m \theta \mathrm{P}-\mathrm{M}=0$. $m \theta \mathrm{R}-i \theta^{2} \mathrm{P}+\mu \mathrm{M}=0,\left(\mathrm{I}_{1} \theta^{2}-h w\right) \mathrm{R}+\mathrm{M}=0$, if
$\mathrm{I}_{1}, \mathrm{I}$ and $\frac{1}{2} i$ are moments of inertia nf the waggnn about the rail, of the $\mathrm{I}_{1}, \mathrm{I}$ and $\frac{1}{2} i$ are moments of inertia nf the waggon abount the rail, of the
frames about $\mathrm{C}$, and of the frame $\mathrm{G}$ about $\mathrm{EJ}$; $\frac{1}{3} \boldsymbol{m}$ is the moment of

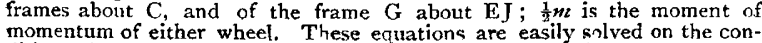
momentum of either wheel. These equations are easily solved on the con-
ditions that at $t=0, \mathrm{R}=\mathrm{R}, \theta, \theta \mathrm{R}=0, \mathrm{P}=0, \theta \mathrm{P}=0$. Assuming no play, that $\mathrm{s}$, that as soon as ' $\mathrm{H}$ leaves $\mathrm{D}, \mathrm{M}^{\prime}$ torches $\mathrm{L}$ ', we can now find the return to the mid-position from a new set of equations. Friction retards the return, it must be remembered. The motions are exceedingly interesting when numerical values of $\mathrm{T}_{1}, \mathrm{I}, i$, \&c., are taken, but the practical man will find $i$ more interesting to make an experimental study of what happens.
Work is here actually done which must be supplied by the electromotors. The restoring moment applied to the car may be made as great as we please by increasing the diameter of $H$. It is true that we cannot in this way alter the total momental impulse, and this is the important thing.

One of the most important things to know is this: the Brennan model is wonderfully successful; the weight of the apparatus is not a large fraction of the weight of the waggon; will this also be the case with a real car which weighs rooo times as much? If at any instant a condition of things is suddenly produced so that the waggon makes an angle $R_{0}$ with its position of equilibrium, if its weight is $W$, its centre of gravity at the height $h$ above the rail, if $I_{1}$ is its moment of inertia about the rail as axis, if $\frac{1}{2} m$ is the moment of momentum of each gyrostat wheel, the momental impulse $m \mathrm{P}_{1}$ ought, roughly, to be equal to $\mathrm{CR}_{0} \sqrt{\mathrm{I}_{1} \mathrm{~W}} h$, where $\mathrm{C}$ is a constant. $I$ use $P_{1}$ for the total angular precession at first. Now the tilt to be corrected, $\mathrm{R}_{0}$, may be due to wind pressure, to a sudden shifting of the centre of gravity, or to centrifugal force, and it is not easy to compare these things in waggons of different sizes. If, however, we take it that the size of each dimension of the waggon is multiplied by $n$ and the size of each dimension of the gyrostatic apparatus is multiplied by $p$, and the wheels have the same peripheral speeds, we find the following results :For wind, $R_{0}$ is proportional to $n^{-1}$. For centrifugal force it seems reasonable to take the speed of a waggon as proportional to $n$, and mean radius of rail curves proportional to $n^{2}$. In this case the result is again that $\mathrm{R}_{0}$ is proportional to $n^{-1}$. As for a possible accidental shifting of the centre of gravity because of the displacement of part of the cargo $w$ through the distance $d$, if we take $w \propto c n^{2}$ and $d<<n$, we find with greater and greater accuracy as $w$ is a smaller fraction of the whole weight, $\mathrm{R}_{0} \propto n^{-1}$.

Taking $P_{1}$, the maximum angle of precession, to be the same in all cases, the above relation leads to the result that $p=n^{\frac{7}{8}}$, or that the fractional weight of the apparatus as compared with that of the waggon is proportional to $W^{-0.125}$, where $W$ is the weight of the waggon. Thus, if we take $n=x 0$, that is, every dimension of the model multiplied by 10 or its weight by I0oo, then $p=7.5$, so that the weight of the gyrostat apparatus is only multiplied by 420 . If in the model the apparatus was io per cent. of the whole weight, in the large waggon the apparatus is only 4 per cent. of the whole weight. In fact, the larger the waggon the less proportion of its weight and volume is occupied by the a.oparatus, a result which must be very satisfactory to Mr. Brennan.

In the cases both of Mr. Schlick and Mr. Brennan, it has to be remembered that if the diameter of the wheel be increased in greater proportion than the dimensions of the ship or waggon, or other dimensions of the wheel, the proportional weight of the apparatus may be diminished. A wheel of twice the diameter, but of the same weight, may have twice the moment of momentum and may be twice as effective. I assume the stresses in the material to be the same.

\section{ON THE PHYSICAL ASPECT OF THE ATOMIC} THEORY. ${ }^{1}$

THE lecture began by setting out a physical reason a priori why matter should be constituted of discrete particles instead of being continuous. The requirements of physics demand an æther to serve as the means of communication between portions of matter out of contact with each other, and space can hardly be conceived as fully occupied simultaneously by two media, matter and æther; hence the matter must be constituted of discrete centres, or nuclei, determining permanent collocations of energy in the æether, which are, in fact, primordial atoms and their fields of force. The feasible problem of atomic physics is to build up an adequate idea of the dynamic constitution of these æthereal fields of force; there is the problem

1 Abstract of the Wilde Lecture of the Manchester Literary and Philo. sophical Society, delivered on March 3 by Prof. J. Larmor, Sec. R.S. 
beyond, to determine the intrinsic constitution of the central nuclei to which they are attached, which may remain permanently beyond our ken. The expansion of our ideas about the atoms, and their structural connection with the æther, was traced from their origin in Descartes, through Huygens and Newton, down to the more definite modern types of representation, as regards various essential features, that are afforded by the vortex atom and the electron.

In the hands of the physicists, especially Newton and Young, the atom had already become a complex structure, capable of definite, inherent, periods of free vibration, but, so far as physics was concerned, the same substance might include various kinds of atoms. The fundamental advance of Dalton, which assured an adequate domain to chemistry as an exact science, was the proof that each compound substance is definite as regards its molecule, and that all atoms of the same elementary body are identical. Whether this absolute identity points to the atom of each chemical element being a dynamically balanced structure of primordial atoms, one of a limited number of possible definite types of structure-which would be a perfectly reasonable way of accounting for this remarkable identity -remains an open question. The periodic relations of the elements, connected most closely with the name of Mendeleeff, certainly indicate that, whatever may be the case as regards the kernel, the outer structure of the atom, so to speak, which is the link through the æther between the nucleus and the outside world, is constituted on the basis of a common ultimate element which may be the electron.

The remark of Maxwell seems still to retain its force, that the mechanism of biological evolution could hardly reside in atoms, primordial or other, which had not much vaster underlying complication than is needed for their purely physical relations. The facts of biology may possibly demand a hypothesis such as the above, that atoms not in intimate contact interact through the æther according to general physical laws, in the manner required to constitute the physical cosmos, but that there may also be a closer interpenetration of atomic nuclei in which far more complex agencies are involved.

The mechanical atom of the earlier physicists, considered in this physical aspect, as an unknown core determining the field of activity in the surrounding æther, has had, since Faraday's discoveries in electrolysis, to take on a more definite form as the electrical atom. The result had been fully reached by Faraday himself, though it needed to be enforced later by Helmholtz, that the energies which have play in chemical combination are of electrical origin implying thereby, according to Maxwell's interpretation, energies of intrinsic stress and motion brought to bear from atomic stores located in the adjacent æther. This doctrine has led on to the modern theory of purely electric atoms, which was already demonstrable on theoretical grounds, of course in a way less definite than we now know it, before the very remarkable discovery of electrons actually free had been reached, through the phenomena of radio-activity either electrically induced or spontaneous. Here again there is the same choice of points of view open to tentative development. We may proceed on a limited hypothesis as if the electrons are the sole primordial atoms; or we may assume that there are various ultimate atoms which have existence and structure of their own, of tvpe largely unknown and independent of the æther, and that the electrons which are associated with them, whether temporarily or intrinsically, form merely one feature of their constitution, viz. their means of communication with the zether, and through it with other atoms at a distance to form an ordered universe.

In any case we are right in following out the hypothesis, there being, in fact, none other open to us, that the purely physical manifestations of atoms--those, namely, that, owing to the simple interconnection involved in their common seat in the æther, aggregate into the definite physical qualities of matter in bulk-are in the main or in most circumstances practically a group by themselves, and that they are thus capable of being investigated on these broad, simple principles of dynamics, which Newton definitely formulated as a suitable foundation for the analysis of general physical activity, as it presents itself in the universe. This so-called mechanical hypothesis has been eminently the fruitful one; it pointed the way to the principle of the conservation of energy, and is now elucidating the wider principle of its definitely limited availability; it gave a rational explanation of the spectrum and of radiation in general, which has proved a trustworthy and precise guide to investigation of phenomena far below the surface, such as the selective dispersion of light and the magnetic action on radiation; it reduced electrical phenomena to order and control, and connected them with light. It must therefore be presumed to be available as the clue for the further elucidation of pressing problems, such as the nature of the transmission of gravitation and of the intimate operation of chemical affinities.

The tendency to reject dynamical analysis as artificial in such subjects as electrodynamics, which received some stimulus from the theoretical writings of Hertz, seems to overlook the fact that it was precisely as a compact working basis suitable for the formulation of experience in its more general aspects that the Newtonian scheme of dynamics was put forward by its author. In the course of time that scheme has become wider and more elastic through the generalisations of Lagrange and Hamilton, expounded forcibly on the physical side by Kelvin, Helmholtz, and various others. But to take over the final results, and dress them in new language devoid of the dynamical implication, seems to involve a misreading of scientific evolution.

This position may be enforced by a quotation from the final exposition of Newton's views on the scope of natural philosophy in general, inserted by himself at the end of the famous "Oueries," in "Opticks," ed. 3, p. 377 :"To tell us that every' Species of Things is endow'd with an occult specifick Quality by which it acts and produces manifest Effects, is to tell us nothing: But to derive two or three general Principles of Motion from Phaenomena, and afterwards to tell us how the Properties and Actions of all corporeal Things follow from those manifest Principles, would be a very great step in Philosophy, though the Causes of those Principles were not yet discovered: And therefore I scruple not to propose the Principles of Motion above mention'd, they being of very general Extent, and leave their Causes to be found out." Then he proceeds to associate-his laws of motion with an atomic theory.

A review of the electrical side of the atomic theory requires a consideration of the phenomena of ionisation in solutions. The theoretical difficulties which have presented themselves in this subject were discussed, in particular the nature of the energy changes which must occur when a salt is dissolved and thus split into separate ions. Reasoning from the processes of the voltaic cell, as expounded after Faraday by Helmholtz, the view is advanced that an equivalent of purely local potential energy of affinity with the solvent must be exhausted in order to provide for the separation of the ions, but without much violent motional disturbance such as would diffuse partially away into the form of heat. This absence of such motional dissipation of the energies of affinity, as indicated, for example, by their almost complete mechanical availability in a Daniell's cell, is perhaps connected with the intimate contacts in confined spaces which are characteristic of the processes at the electrodes by which the chemical change is effected. It is suggested that a similar mode of explanation applies to the very high, sometimes nearly complete, mechanical availability (Berthelot) of the energy of chemical transformations in dense media such as liquids and solids, as contrasted with dilute systems such as gases, which the recent work of Nernst and his pupils has brought again to the front.

The lecture passes on to touch on those extensive branches of chemical physics to which the constitution of the atom is not essential, where only a statistical grasp of the molecular associations and dissociations that are taking place is required. The quantitative theory of chemical equilibrium and of progress of chemical change as regards dilute systems comes under this head, of which the prototype and the most highly developed example is the kinetic theory of gases. The modern theory of electrodynamics, as based on the displacements and motions of electrons, is in the main analogous, and the theory of 
gravitation, when it comes to light, will be of the saune kind.

In particular, the molecular aspect of reaction in gases is passed under review. Reasons are brought forward for holding that in gases all ultimate reactions are of necessity mono- or bi-molecular. If this be so, the important work now proceeding with regard to the effect of impurities in promoting or inhibiting gaseous reactions must lead to fuller knowledge of the transient molecules or radicals which are formed in the destructive encounter of a pair of the reacting molecules, and are the carriers or intermediaries leading finally to poly-molecular change; while the same transient combinations may be approachable independently from another side as affording. the interpretation of the complex banded spectra of emission or absorption in gaseous media.

The very remarkable and most fruitful and prophetic symbolic theories of molecular structure, especially for the complex molecules of organic chemistry, have not yet proved capable of dynamical interpretation; it seems necessary, however, to admit, on account of the wide range of physical properties that are nearly atomically additive, that stereochemical collocations do represent in some real way the actual aggregation of the atoms instead of mere symbolical representation of it. Recent investigation appears to bring out in certain cases a somewhat definite relation between the configuration of the molecule and the crystalline form of its physical aggregations, which, though reasonable, could not have been foreseen a priori; exact crystallographic measurements may thus in time afford another intimate clue to the molecular structures in related series of compounds.

\section{A NEW METHOD OF STEREOSCOPIC PHOTOGRAPHY.}

A $\mathrm{N}$ entirely novel suggestion for the production of stereoscopic photographs is proposed by Prof. G. Lippmann in the current number of the Comptes rendus of the Paris Academy of Sciences (March 2). Let a lens be constructed of a material possessing a refractive index $n$, the segments forming the front and back of the lens having the same centre of curvature and the ratio of the radius of curvature of the front segment to that at the back being $n-\mathrm{r}$. The front surface is the receiving lens, and corresponds to the lens of the eye; the back surface is covered with the sensitive emulsion, and corresponds to the retina, Owing to the chosen relation between the curvatures of the two faces an image of a point is formed by the front surface on the back one. The system is reversible; a ray of light proceeding from any point of the receiving surface will pass out at the front over exactly the same path as that taken by the incoming light in acting on the sensitive film, and this will be true in spite of any imperfection of the lens surfaces.

Prof. Lippmann now imagines a material such as celluloid moulded back and front, so that the whole surface is covered with microscopic cells, each of which is an elementary cell possessing the properties of the single lens described above. The whole film resembles the compound eye of insects. This plate, sensitised, is exposed in full daylight to the objects to be represented, no photographic lens being required. The result of the operation would be a series of microscopic images fixed each on the "retina" of one of the cells. Seen from the side of the sensitive layer, the whole plate would present a uniformly grey appearance. But seen from the front and illuminated from behind the plate (supposed converted into a positive), the photograph would appear, and would possess the following peculiarities. It would appear in true relief, exactly as in nature, and shifting the eye about would produce a change in the photograph seen, the effect being as if the observer stood in front of a window. By stepping from side to side, in the latter case, fresh portions of the landscape would come into view, the whole always being bounded bv the four sides of the window. In the case of such a piate as that described, the effect would be precisely similar.

As the author remarks in the paper, the technical difficultirs in the prevaration of such a plate would be verv great. The chief difficulty would be the fulfilment of the con- dition necessary for the clear definition of each image in each elementary cell; the ratio, of the radii of curvature must be equal to $n-\mathrm{r}$. Considering the thickness of the film, this difficulty would appear to be insuperable, but it is to be hoped that an attempt will be made to put this idea into practice, however imperfectly.

\section{UNIVERSITY AND EDUCATIONAL INTELLIGENCE.}

CAMBRIDGE.-During the last four years the average income of the botanic garden has been $1708 l$., to which the University chest has contributed 1175l., the rest being made up of money from trust funds and from rents. The botanic garden syndicate now points out that the income is no longer sufficient to cover the expenses. The syndicate estimates that in future, if the gardens are to maintain their high position amongst kindred institutions, some additional income must be found, and it is recommended that the present grant from the University chest to the botanic garden be increased by the sum of $220 l$. per annum.

The next combined examination for sixty-seven entrance scholarships and a large number of exhibitions at Pem. broke, Gonville and Carus, King's, Jesus, Christ's, St. John's, and Emmanuel Colleges will be held on Tuesday, December I, and following days. Mathematics, classics, and natural sciences will be the subjects of examination at all these colleges. Some of the colleges allow candidates who intend to study mechanical sciences to compete for scholarships and exhibitions by taking the papers set in mathematics or natural science. The colleges desire it to be known that any candidate for a scholarship may signify in writing his wish nat to receive the emolument of the same if elected thereto, and that such candidate may be elected to a scholarship which may be honorary only and without emolument, but shall carry with it all other privileges attached to the position of a scholar. The amount thus set free will serve to increase the number of scholarships or exhibitions open to other candidates.

GLASGOW.-Among the honorary degrees to be conferred by the University on April 22 are the following :-LL.D.: Mr. G. T. Beilby, F.R.S., chairman of the governors of Glasgow and West of Scotland Technical College; Colonel David Bruce, C.B.; F.R.S.; Dr. J. J. Dobbie, F.R.S., director of the Royal Scottish Museum, Edinburgh; Mr. R. Kidston, F.R.S.; and Dr.J. C. McVail, county medical officer, Stirlingshire and Dumbartonshire.

Dr. R. Stewart MacDougall, on his appointment to the lectureship in botany in Edinburgh University, has resigned his position as biologist on the staff of the Edinburgh and East of Scotland College of Agriculture.

Mr. A. I. Bowley, reader in statistics in the University of London, will give a course of ten lectures on elementary applications of mathematics to statistical data at the School of Economics, at 7 p.m., on Thursdays, March 26 and April 2, resuming after the Easter vacation on May 7 , and continuing thereafter for seven consecutive Thursdays.

THE Earl of Rosebery will visit University College on the afternoon of Thursday, March 26, and will formally open the new libraries and the new south wing, which includes lecture-rooms for the faculty of arts, the departments of geology, hygiene, and experimental psychology, also large extensions of the departments of applied mathematics, of mechanical, electrical, and municipal engineer ing, and accommodation for the new hydraulic laboratory.

THE first volume of the report of the U.S. Commissioner of Education for the year ending June 30, 1906, has been received from Washington. In addition to chapters summarising the progress made during the year under review in the various departments of American education, the report contains a series of excellent articles on educational administration in various European and other countries. A useful summary of the different sections of the report is provided in the commissioner's introduction, 